ARTICLE

DOI: $10.1038 /$ s41467-018-06051-1

\title{
Nucleolar fibrillarin is an evolutionarily conserved regulator of bacterial pathogen resistance
}

\author{
Varnesh Tiku²,2, ${ }^{1,2}$ Chun Kew ${ }^{1}$, Parul Mehrotra ${ }^{1,5}$, Raja Ganesan ${ }^{2,3}$, Nirmal Robinson ${ }^{2,6}$ \& Adam Antebi ${ }^{1,2}$
}

Innate immunity is the first line of defense against infections. Pathways regulating innate responses can also modulate other processes, including stress resistance and longevity. Increasing evidence suggests a role for the nucleolus in regulating cellular processes implicated in health and disease. Here we show the highly conserved nucleolar protein, fibrillarin, is a vital factor regulating pathogen resistance. Fibrillarin knockdown enhances resistance in C. elegans against bacterial pathogens, higher levels of fibrillarin induce susceptibility to infection. Pathogenic infection reduces nucleolar size, ribsosomal RNA, and fibrillarin levels. Genetic epistasis reveals fibrillarin functions independently of the major innate immunity mediators, suggesting novel mechanisms of pathogen resistance. Bacterial infection also reduces nucleolar size and fibrillarin levels in mammalian cells. Fibrillarin knockdown prior to infection increases intracellular bacterial clearance, reduces inflammation, and enhances cell survival. Collectively, these findings reveal an evolutionarily conserved role of fibrillarin in infection resistance and suggest the nucleolus as a focal point in innate immune responses.

\footnotetext{
${ }^{1}$ Max Planck Institute for Biology of Ageing, Joseph Stelzmann Strasse 9b, 50931 Cologne, Germany. ${ }^{2}$ Cologne Excellence Cluster on Cellular Stress Responses in Aging-Associated Diseases (CECAD), University of Cologne, 50674 Cologne, Germany. ${ }^{3}$ Institute for Medical Microbiology, Immunology and Hygiene, University of Cologne, 50674 Cologne, Germany. ${ }^{4}$ Present address: Department of Infectious Diseases, Genentech Inc., 1 DNA Way, South San Francisco, CA 94080, USA. ${ }^{5}$ Present address: VIB-Center for Inflammation Research, VIB - Ghent University, Technologiepark 927,9052 Ghent, Belgium. ${ }^{6}$ Present address: Centre for Cancer Biology, University of South Australia, HB11-35 UniSA CRI Building, North Terrace, 5001 Adelaide, Australia. These authors contributed equally: Varnesh Tiku, Chun Kew, Parul Mehrotra. These authors jointly supervised this work: Nirmal Robinson, Adam Antebi. Correspondence and requests for materials should be addressed to N.R. (email: nirmal.robinson@uk-koeln.de) or to A.A. (email: Antebi@age.mpg.de)
} 
nnate immunity helps combat infections in the absence of prior exposure to pathogens. Pathogen recognition by the host entails detection of characteristic molecular patterns associated with the pathogen, so-called pathogen-associated molecular patterns (PAMPs) ${ }^{1}$. The host uses specialized factors called pattern recognition receptors (PRRs) to detect PAMPs triggering an immune response, which involves upregulation of immune response genes. However, PAMPs are associated with a wide range of microbes, both pathogenic and commensal. Therefore, the question arises how does an organism distinguish between a pathogenic and a non-pathogenic microbe if it is only reliant on the PAMPs detection system.

A relatively new concept in the field of innate immunity is the so-called effector-triggered immunity, whereby host cells are alerted to the pathogen by the associated damage caused by pathogen secreted toxins or virulence factors ${ }^{2,3}$. For instance, virulent toxins produced by diverse pathogens including Pseudomonas aeruginosa ${ }^{4}$, Legionella pneumophila ${ }^{5}$, and Shiga toxinsecreting Escherichia coli ${ }^{6,7}$ often suppress host messenger RNA (mRNA) translation to inhibit expression of anti-microbial factors and thereby assist in bacterial survival ${ }^{8}$. Many of these toxins have different conformational properties and it would be inefficient for the host to have specific PRRs capable of recognizing all these diverse structures. Therefore, nature has employed a rather efficient way of pathogen detection by encountering the damage caused by the pathogen to the host. This theme of effectortriggered immunity has been well established in plants 9,10 and more recently has been appreciated in animal models including Caenorhabditis elegans ${ }^{11}$.

The nucleolus is best known as a sub-nuclear organelle where ribosomal RNA (rRNA) is synthesized and assembled into ribosomal subunits. Recent evidence, however, also suggests that the nucleolus aids in the assembly of other ribonucleoprotein particles, including splicing factors, signal recognition particle, small interfering RNA (siRNA) machinery, stress granules, and telomerase $\mathrm{e}^{12}$, and regulates physiologic processes such as stress responses $^{13}$ and aging ${ }^{14,15}$. Interestingly, a handful of studies have linked the nucleolus with viral infection. Several viral proteins localize in the nucleolus after infection ${ }^{16}$, and viruses subvert the host cell by recruiting nucleolar proteins for viral replication ${ }^{16}$. However, the potential of this organelle in innate immunity in bacterial infections remains largely unexplored.

Here we report that downregulation of the highly conserved nucleolar protein fibrillarin increases infection resistance of $C$. elegans against the bacterial pathogens Staphylococcus aureus, Enterococcus faecalis, and $P$. aeruginosa. We show that bacterial infection leads to a reduction in nucleolar size, rRNA, and fibrillarin levels. Fibrillarin knockdown confers resistance to the known infection-sensitive mutants, suggesting that fibrillarin reduction-mediated protection is independent of the major immune response pathways in C. elegans. Fibrillarin levels are also reduced upon infection in mammalian cells and its prior knockdown enhanced clearance of intracellular bacteria, improved cell survival, and reduced inflammation after infection. We propose that fibrillarin acts as a central node in a regulatory network engaged in imparting immunity against bacterial pathogens, conserved across evolution.

\section{Results}

Fibrillarin regulates resistance to bacterial infection. C. elegans $f i b-1$ encodes the highly conserved nucleolar methyltransferase fibrillarin, which is a vital factor in the $\mathrm{C} / \mathrm{D}$ small nucleolar ribonucleoprotein (snoRNP) complex. Fibrillarin mediates $2^{\prime}$-Oribose methylation of rRNA thereby assisting in the maturation of rRNA $^{17,18}$, and also methylates histone H2AGln105 at the rDNA locus ${ }^{19}$. We recently reported that $f i b-1$ is downregulated in multiple well-established longevity mutants of $C$. elegans and that fib-1 knockdown reduces nucleolar size and extends lifespan in worms ${ }^{14}$. Since genes that promote lifespan extension often induce tolerance against multiple stress conditions including pathogenic infections ${ }^{20-22}$, we wondered if fib-1 reduction could confer infection resistance against pathogens. We knocked-down fib-1 using RNAi and monitored infection resistance in worms. Since $f i b-1$ is an essential gene, we resorted to RNAi from larval stage 3 (L3) up to day one of adulthood (around $30 \mathrm{~h}$ of fib-1 RNAi), which led to a significant reduction in FIB-1 levels without causing any developmental defects (Supplementary Fig. 1A). Interestingly, animals with fib-1 RNAi displayed significantly increased survival upon infection with pathogens $S$. aureus, E. faecalis, and $P$. aeruginosa (Fig. 1a, b and Supplementary Fig 1B). fib-1 knockdown did not affect other stress responses including heat, cold, and oxidative stress resistance, suggesting that $f i b-1$ specifically regulates pathogen resistance (Supplementary Fig 1C-E). fib-1 RNAi did not affect pharyngeal pumping rate, ruling out the possibility of differences in bacterial intake (Supplementary Fig. 1F). Reduction in major cellular processes is known to induce food aversion behavior in C. elegans, which has been linked to innate immunity ${ }^{23}$. However, fib-1 knockdown-mediated improvement in infection resistance was independent of food aversion behavior (Supplementary Fig. 1G, $\mathrm{H})$. We and others have shown previously the B-box protein NCL-1/TRIM2 to be an upstream negative regulator of FIB$1^{14,24,25}$. FIB-1 levels are highly upregulated in ncl-1 loss-offunction mutants. Therefore, we tested the survival of $n \mathrm{cl}-1$ mutants upon infection. Interestingly, we found ncl-1 mutants were more susceptible to infection, suggesting that increased levels of fib-1 are detrimental for survival upon infection challenge (Fig. 1c, d). Taken together, our results reveal a state of protection conferred by fib-1 reduction that helps worms survive longer upon infection.

Fibrillarin and nucleolar size are reduced upon infection. Next, we assessed the levels of FIB-1 after infection. We performed western blot to detect the endogenous levels of FIB-1 after 12-h infection in wild-type and more susceptible ncl-1 mutants. We observed a downregulation of FIB-1 protein levels in wild-type worms after infection with $S$. aureus, E. faecalis, and P. aeruginosa (Fig. 2a, b, Supplementary Fig. 2A). ncl-1 mutants also exhibited a slight reduction of FIB-1 after infection, but the levels remained significantly higher compared to wild type (Fig. 2a, b). We also obtained similar results with the FIB-1::GFP strain harboring a translational fusion; the GFP signal was significantly downregulated after infection with $S$. aureus (Fig. 2c). Notably, we did not observe significant transcriptional changes of fib-1 using qPCR, suggesting a post-transcriptional response (Supplementary Fig. 2B). In our previous study, we reported that the nucleolar size decreased in worms subjected to fib-1 RNAi ${ }^{14}$. Because we observed a reduction in FIB-1 levels after infection, we wondered if nucleolar size changes correspondingly. Indeed, we observed a significant decrease ( $25 \%)$ in the nucleolar size of worms after 12-h infection with $S$. aureus and E. faecalis (Fig. $2 \mathrm{~d}$, e). The size of nucleoli did not change when worms were fed heat-killed $S$. aureus and E. faecalis, suggesting that the nucleolar size reduction was caused by active infection (Supplementary Fig. 2C, D). Since the nucleolus is the site of rRNA maturation and FIB-1 plays a crucial role in this process, we wondered if rRNA levels were altered after infection. As predicted, infection with $S$. aureus led to a reduction of mature rRNA levels (Fig. 2f). $n c l-1(+)$ is known to limit nucleolar size in worms; $n c l-1$ null mutants possess enlarged nucleoli in multiple tissues ${ }^{26,27}$. We assessed the 
a

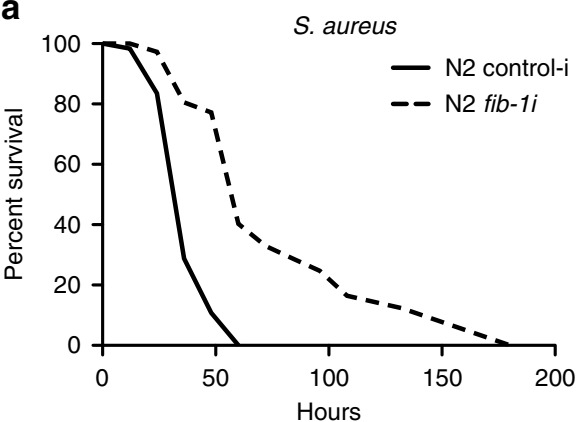

C

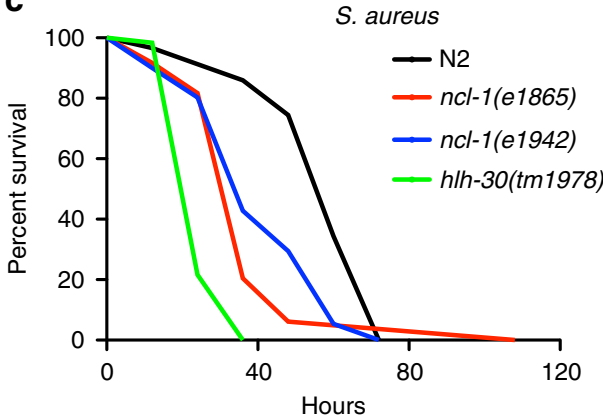

b

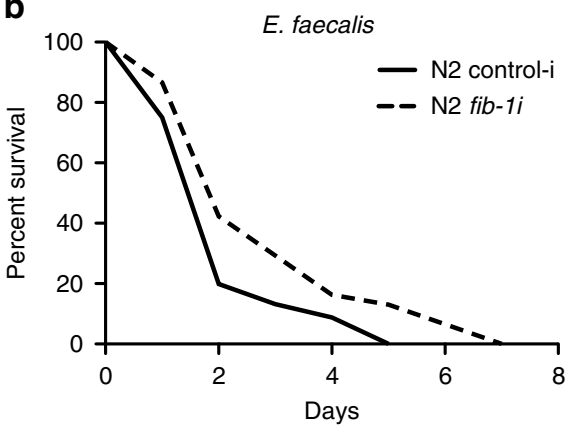

d

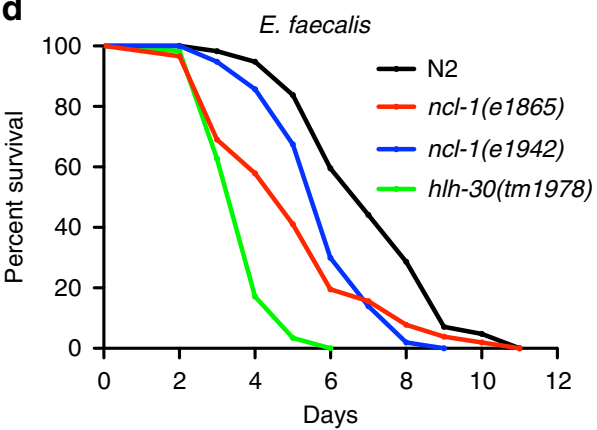

Fig. 1 fib-1/fibrillarin regulates bacterial infection resistance in C. elegans. $\mathbf{a}, \mathbf{b}$ fib-1 knockdown improves survival of wild-type N2 worms upon S. aureus and E. faecalis infection $(P<0.0001)$. c, d ncl-1 mutants (e1865 and e1942) are short-lived compared to wild-type N2 upon infection with S. aureus and E. faecalis $(P<0.0001)$. hlh-30(tm1978) served as a control for infection. Survival experiments were performed three times independently. $P$-values were calculated by log-rank test

nucleolar size of $n c l-1$ mutants after infection and observed that unlike wild-type worms, nucleolar size remained enlarged (Fig. 2d, e). Taken together, these results suggest that a reduction in FIB-1 levels and nucleolar size might be a host response toward combating infection challenge. ncl-1 mutants are somewhat refractory in this response, which might explain their increased susceptibility.

Fibrillarin affects survival of pathogen sensitive mutants. We next sought to investigate the link between fib-1 and established major defense-response pathways in C. elegans. We examined genetic epistasis between $f i b-1$ and known vital mediators of defense-response upon pathogenic insult in worms. p38 MAP kinase (MAPK) pathway is a key evolutionarily conserved defense-response pathway that is activated upon microbial infection and mediates important downstream transcriptional changes ${ }^{28}$. p38 MAPK, which is encoded by pmk-1 in C. elegans, is the major regulator of this pathway ${ }^{29}$. A mutation in pmk-1 leads to increased susceptibility of worms upon infection with diverse pathogens. fib-1 knockdown significantly improved the survival of pmk-1 (Fig. 3a, Supplementary Fig. 3A). Next we tested $h l h-30 /$ TFEB, a crucial factor promoting autophagy and anti-microbial gene expression as part of the host response upon infection in worms and mammals ${ }^{30}$. Previous reports have shown that HLH-30/TFEB is nuclear localized upon bacterial infection and is required for activation of autophagy and anti-microbial gene transcription in C. elegans ${ }^{30,31}$. However, fib-1 knockdown did not affect HLH-30 nuclear localization (Supplementary Fig. 4A, B). Interestingly, knocking down fib-1 in hlh-30 mutants also led to a significant increase in survival of these animals upon infection (Fig. 3b, Supplementary Fig. 3A). fib-1 RNAi did not affect autophagic flux under uninfected conditions, as measured by the cleavage of the autophagosomal membrane protein LC3/ LGG-1, although under starvation and infection autophagic flux increased as expected (Supplementary Fig. 4C). These findings suggest that the protective effects imparted by fib-1 reduction function independently of HLH-30/TFEB. Another proteostasis mechanism, the ubiquitin-proteasome system (UPS) was also examined upon fib-1 knockdown and $S$. aureus infection. Total levels of ubiquitinated proteins remained similar in control and fib-1 RNAi animals and there was also no noticeable difference upon infection, suggesting that the enhanced survival conferred by fib-1 RNAi does not obviously arise from increased proteolytic mechanisms (Supplementary Fig 4D). Finally we examined the epistasis with daf-16/FOXO, another important highly conserved transcription factor driving anti-microbial genes ${ }^{32,33}$ and infection resistance of $d a f-2$ mutants ${ }^{21}$. Similar to the effects on $p m k-1$ and $h l h-30$, daf-16 showed increased survival on infection upon fib-1 RNAi (Fig. 3c, Supplementary Fig. 3A). Furthermore, reduced survival of $n c l-1$ mutants was also rescued with $f i b-1$ RNAi (Fig. 3d, Supplementary Fig. 3B, C). Taken together, our results reveal FIB-1 as a novel regulator of host response toward infection working downstream or independently of established defense-response pathways in worms.

Fibrillarin reduction induces translation suppression. To investigate the mechanism behind fib-1 reduction-mediated pathogen resistance, we studied the involvement of mRNA translation. Recent studies have reported that worms detect translation suppression by infection as a means to activate defense response ${ }^{34,35}$. Since FIB-1 is a methytransferase involved in rRNA maturation and ribosome biogenesis, we hypothesized that fib-1 RNAi might lead to a reduction in ribosome biogenesis and thereby translation, thus activating the defense response. To test this possibility, we examined a well-established reporter gene, irg-1 (infection response gene-1) fused to GFP. $\operatorname{irg}-1$ is activated by the bZIP transcription factor ZIP-2 during pathogen challenge. In response to a block in translation upon infection with $P$. aeruginosa, ZIP-2 itself is preferentially translated in a manner dependent upon its upstream $5^{\prime}-\mathrm{UTR}^{36}$. After infection with 
a

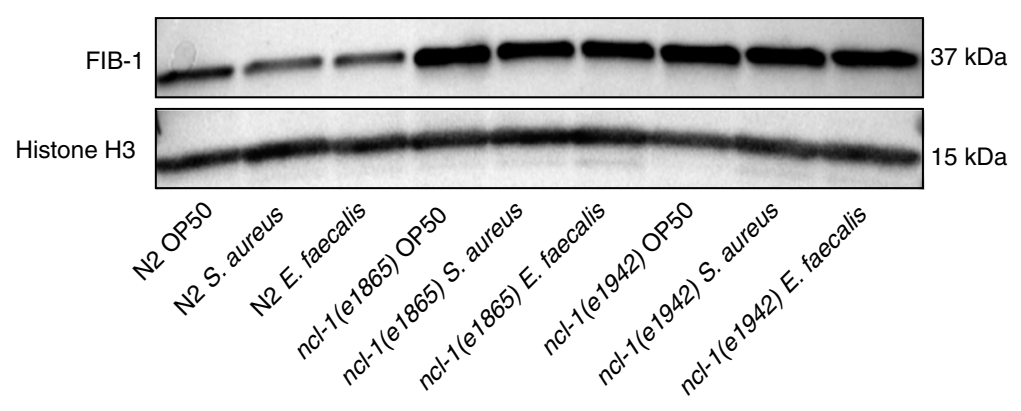

b

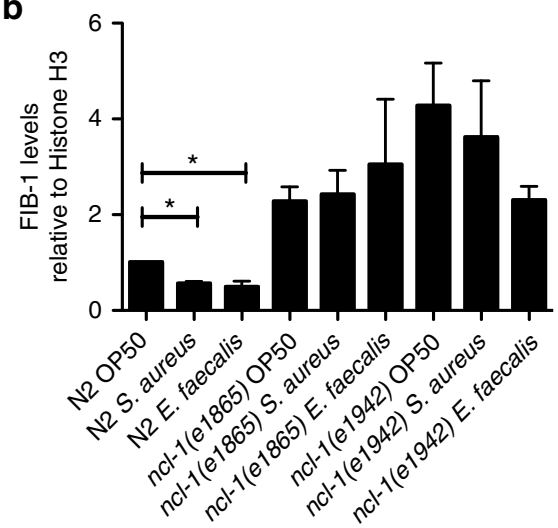

C

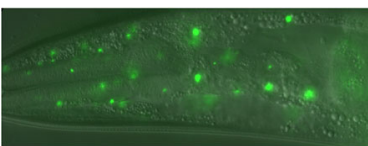

N2+FIB-1::GFP (OP50)

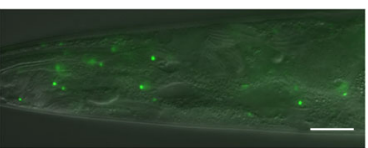

N2+FIB-1::GFP (S. aureus) d
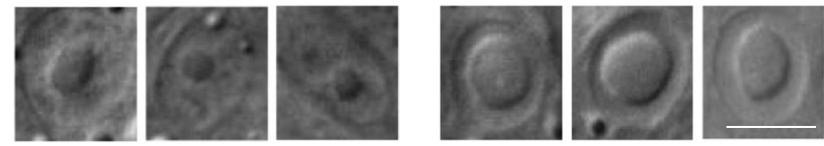

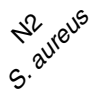

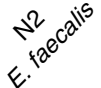

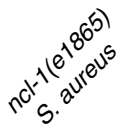

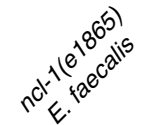

e

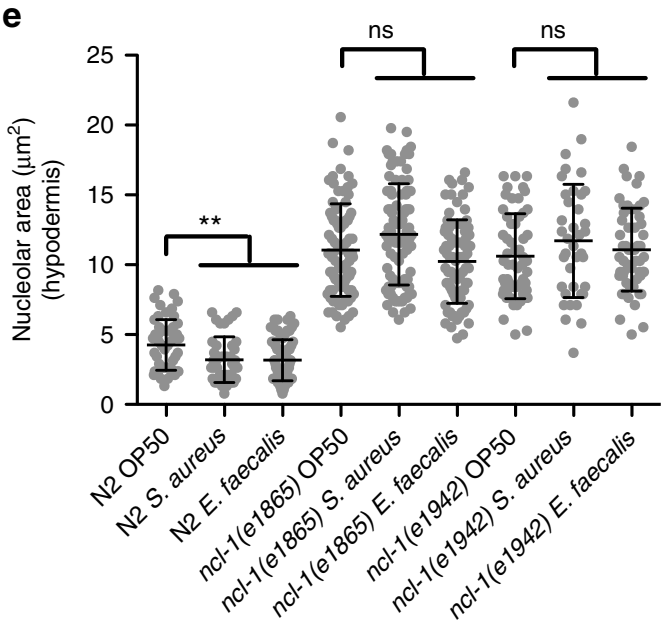

f

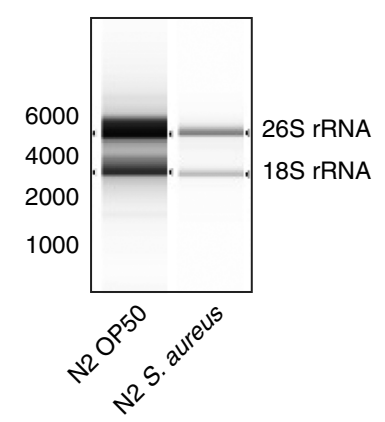

Fig. 2 FIB-1/fibrillarin and nucleolar size are reduced upon bacterial infection. a, b FIB-1 levels are significantly reduced in wild-type N2 after S. aureus and $E$. faecalis infection. FIB-1 is modestly reduced in ncl-1 mutants after infection but the levels are still higher relative to wild-type N2 after infection. Error bars represent mean \pm s.e.m. of three independent biological replicates. c FIB-1::GFP shows reduced fluorescent signal after infection with S. aureus. d, e Nucleolar size in hypodermal cells of wild-type N2 worms is reduced after 12-h infection with S. aureus and E. faecalis. Nucleolar size in hypodermal cells of $n \mathrm{l}-1$ mutants after infection remains unaffected. Error bars represent mean $\pm \mathrm{s}$.d. $\mathbf{f} \mathrm{S}$. aureus infection for $12 \mathrm{~h}$ reduces $26 \mathrm{~S}$ and $18 \mathrm{~S}$ rRNA levels in wildtype $C$. elegans. RNA extracted from equal number of uninfected and infected worms was analyzed using a bioanalyzer. Scale bars represent 20 (c) and $5 \mu \mathrm{m}(\mathbf{d}) .{ }^{\star} P<0.05,{ }^{\star \star} P<0.01$, ns-non-significant, unpaired $t$-test

S. aureus, we observed a strong induction of $\operatorname{irg}-1$ (Fig. $4 \mathrm{a}, \mathrm{b})$. Interestingly, we observed a similar induction of $\operatorname{irg}-1$ transgene as well as the $\operatorname{irg}-1$ transcript upon fib-1 knockdown without infection, suggesting that fib-1 RNAi might reduce translation and thereby activate $\operatorname{irg}-1$ (Fig. $4 \mathrm{c}-\mathrm{e}$ ). As a control, the translational inhibitor cycloheximide also induced $i r g-1$ in a similar manner (Supplementary Fig. 5A). This was not a generalized inflammatory response, however, as other major infection related genes did not change upon fib-1 RNAi (Supplementary Fig. 5B). Consistent with reduced translation, fib-1 RNAi led to a reduction in mature rRNA levels (Fig. 4f). Additionally, fib-1 knockdown also attenuated translation as suggested by a reduction in puromycin incorporation (Fig. 4g, h). We also observed an increase in the precursor-rRNA (pre-rRNA) levels upon fib-1 knockdown (Supplementary Fig. 5C). Conceivably, this pre-rRNA species accumulates due to a block in the maturation process by reduced FIB-1 levels. These data also suggest that the decrease of mature rRNA levels in fib-1 knockdown animals occurs posttranscriptionally.

Since we observed effects of fib-1 reduction on rRNA levels and translation, we next sought to test if fib-1-mediated protection is mediated by a reduction in translation. To address this question, we first asked if mutants with reduced translation have improved infection resistance. We used ifg- 1 and ife- 2 mutants, which are known to have reduced translation ${ }^{37-39}$. ifg- 1 encodes the translation initiation factor eIF4G, while ife- 2 encodes the cap binding initiation factor eIF4E. Indeed ifg- 1 and ife- 2 strains were significantly resistant to infection (Fig. 4i, Supplementary Fig. 5D). Moreover, fib-1 RNAi only marginally enhanced the survival of ifg- 1 mutants upon infection (Fig. $4 \mathrm{j}, \mathrm{k}$ ). These results collectively 

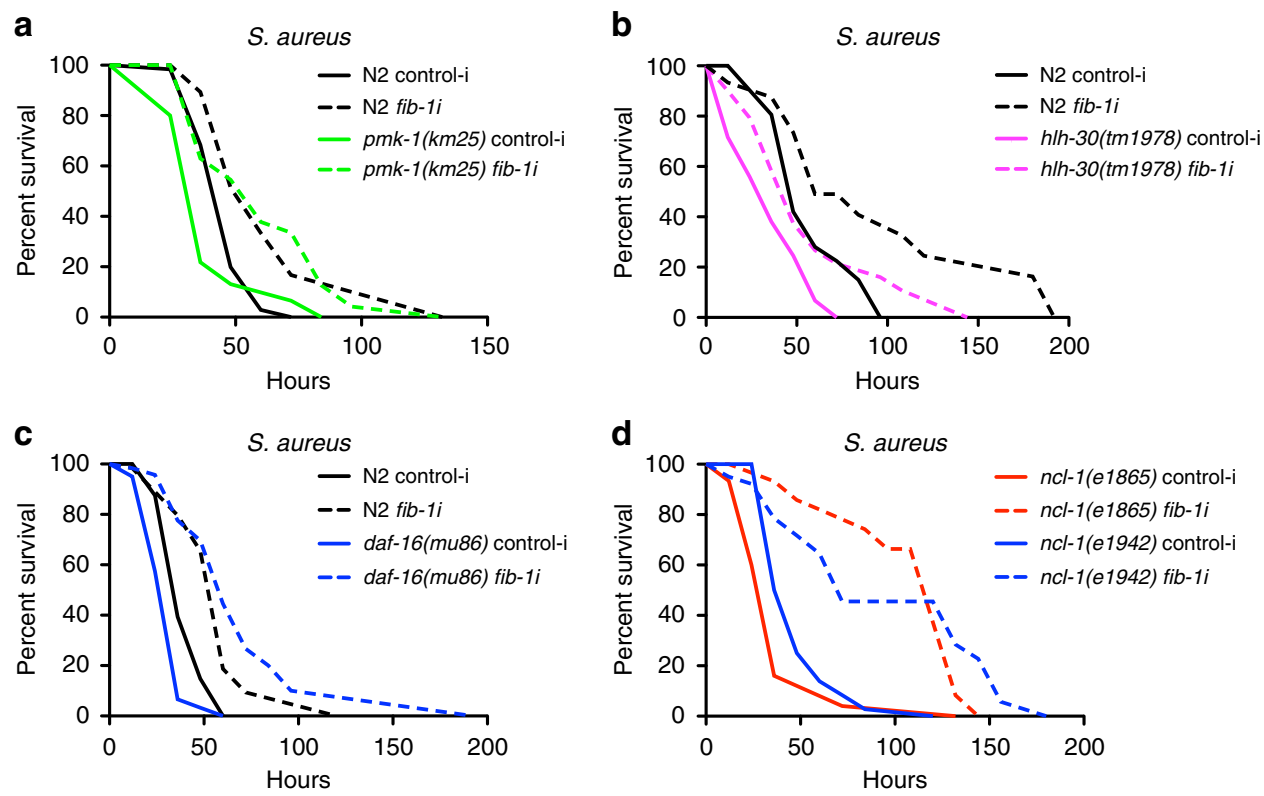

Fig. 3 fib-1/fibrillarin reduction improves resistance of infection-sensitive mutants. a-d fib-1 RNAi significantly improves the survival of infection-sensitive pmk-1(km25) $(P=0.0001)$, hlh-30(tm1978) $(P=0.0021)$, daf-16(mu86) $(P<0.0001)$ and $n c l-1(e 1865$ and e1942) mutants $(P<0.0001)$ upon $S$. aureus infection. Survival experiments were performed three times independently. $P$-values were calculated by log-rank test

suggest that fib-1 knockdown-mediated pathogen resistance mechanistically overlaps with infection resistance conferred by translational reduction.

Fibrillarin reduction protects mammalian cells. We next sought to understand the potential role of fibrillarin in imparting immunity against infections in mammalian systems. To begin with, we wondered if mammalian fibrillarin levels are also perturbed after infection, as observed in C. elegans. We found that HeLa cells infected with $S$. aureus exhibited significantly reduced levels of fibrillarin at varying multiplicities of infections (MOIs) (Fig. 5a). Similarly mouse bone marrow-derived macrophages infected with $S$. aureus, E. faecalis, Salmonella typhimurium, and Listeria monocytogenes showed reduced levels of fibrillarin after $24 \mathrm{~h}$ of infection (Fig. 5b, c). Moreover, we also observed a modest reduction in nucleolar size in THP1 cells $24 \mathrm{~h}$ post infection with $S$. aureus similar to our results in worms, indicating that nucleolar size reduction is a conserved host response to infection (Fig. 5d, e).

We next wondered whether fibrillarin reduction post infection in mammalian systems is protective. We reasoned that if fibrillarin was reduced before infection, it could prime a host response to incoming pathogens. Using macrophages and HeLa cells, we investigated the role of fibrillarin in regulating infection resistance in the mammalian system. A major feature of $S$. aureus infection is the ability of the pathogen to induce inflammation and host-cell death, a phenomenon attributed to the pathogenicity of the bacteria ${ }^{40}$. We performed siRNA-mediated silencing of fibrillarin $48 \mathrm{~h}$ before infection in HeLa cells (Supplementary Fig. 6A) and compared the percentage of cells surviving at 6 and $24 \mathrm{~h}$ post infection in both fibrillarin and control siRNA-treated cells. Fibrillarin knockdown cells displayed significantly better survival after infection with $S$. aureus compared to control siRNA-treated cells as assayed by lactate dehydrogenase (LDH) cytotoxicity assay and trypan blue staining (Supplementary Fig. 6B, C). Murine bone marrow-derived macrophages also exhibited significantly reduced cytotoxicity after treatment with fibrillarin siRNA (Fig. 5f). Interestingly, fibrillarin knockdown improved clearance of intracellular $S$. aureus in murine bone marrow-derived macrophages (Fig. 5g), which possibly explains the augmented resistance in these cells to infection upon fibrillarin knockdown. Conversely, fibrillarin overexpression modestly enhanced the susceptibility of HeLa cells to infection (Supplementary Fig. 6D). Fibrillarin knockdown and overexpression did not influence bacterial uptake by cells as measured by comparing intracellular colony forming units (CFU) with respective controls (Supplementary Fig. 6E, F) ruling out the possibility of differences in bacterial internalization with fibrillarin siRNA. Furthermore, fibrillarin knockdown prior to infection led to a reduction of pro-inflammatory cytokines interleukin (IL)-6 and IL-8 (Fig. 5h), and an induction of antiinflammatory cytokine IL-10 (Fig. 5i) indicative of reduced inflammation. Immunofluorescence using GFP-labeled S. aureus showed increased apposition of the intracellular bacteria to lysosomes in cells treated with fibrillarin siRNA compared to control siRNA (Fig. 5j, k), which might explain increased intracellular bacterial clearance and enhanced cell survival upon fibrillarin knockdown. Taken together, these results point toward a conserved mechanism of bacterial infection resistance possibly imparted by increased phagosome maturation and reduced inflammation that is mediated by fibrillarin knockdown in mammalian cells.

\section{Discussion}

Perturbation of biological systems by infection leads to a multilayer complex cellular and organismal response. Whether this response ultimately leads to clearance of infection or collapse of the host system is largely dependent on the extent and nature of the cellular pathways perturbed and the interplay between the host and the pathogen.

Cellular organelles such as mitochondria, endoplasmic reticulum (ER), and lysosome have long been identified as signaling hubs that help manage infection. However, a role for the nucleolus in mediating the innate response to pathogenic stress is relatively unstudied. In this work, we identified the nucleolar protein fibrillarin as a novel player in regulating bacterial pathogen resistance of $C$. elegans. Fibrillarin reduction increases the survival of worms challenged with S. aureus, E. faecalis, and 


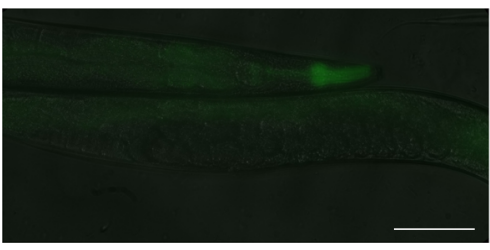

$\mathrm{N} 2+\mathrm{P}_{\text {irg- } 1}$ GFP OP50

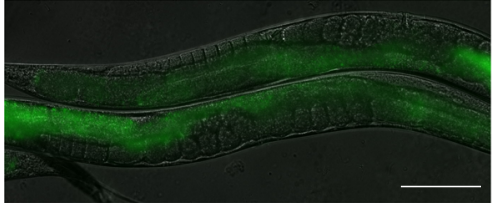

$\mathrm{N} 2+\mathrm{P}_{\text {irg-1 }}$ GFP control-i

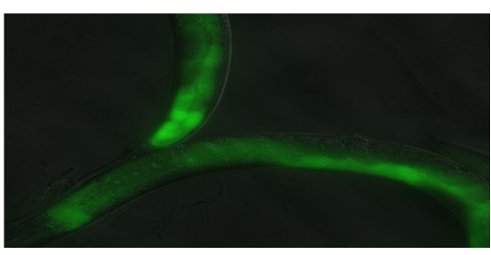

$\mathrm{N} 2+\mathrm{P}_{\text {irg-1 }}$ GFP S. aureus

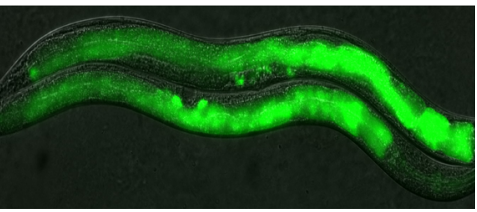

$\mathrm{N} 2+\mathrm{P}_{\text {irg-1 }}$ GFP fib-1i b

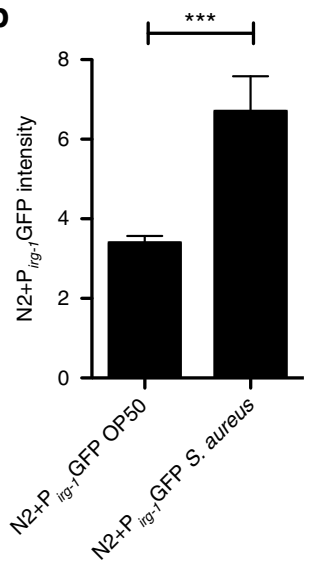

d

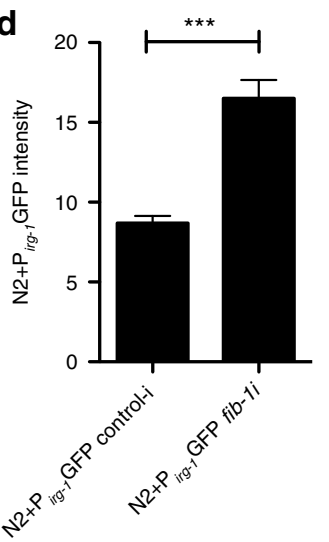

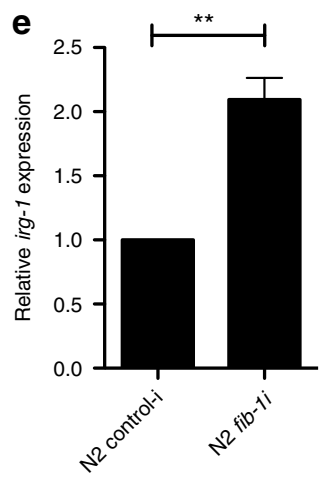

f

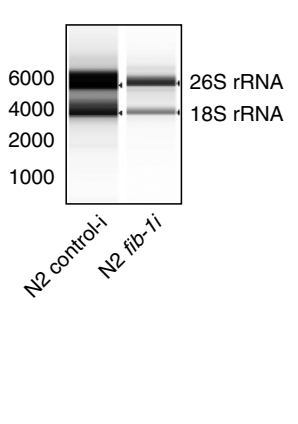

g

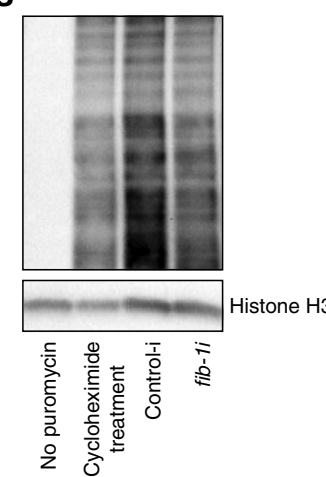

h

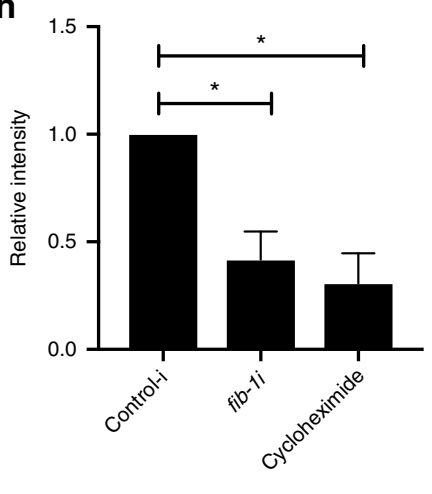

i

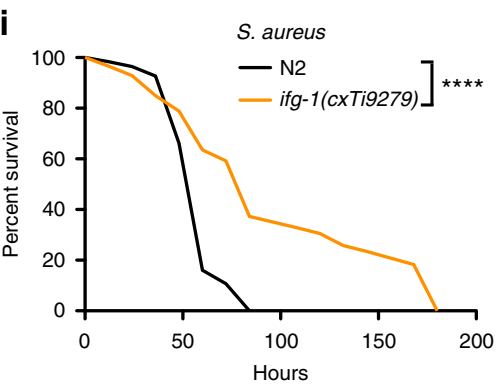

j

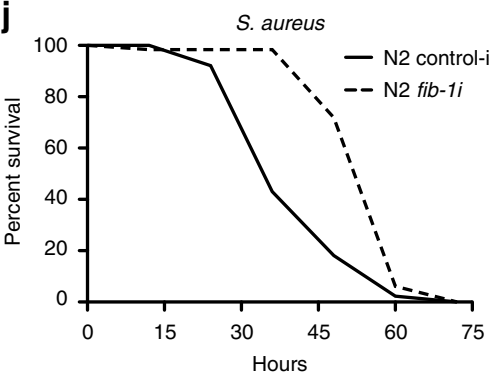

k

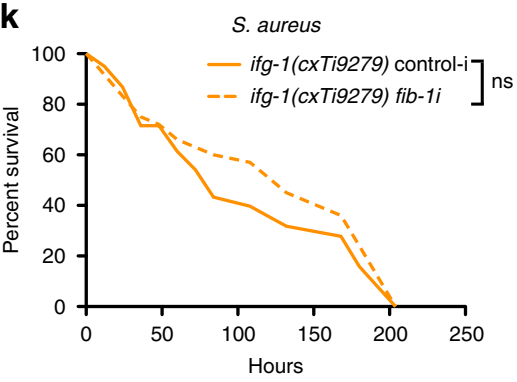

Fig. 4 fib-1/fibrillarin reduction induces translation suppression. a, b Twelve-hour $S$. aureus infection induces $P_{\text {irg-1 }} G F P$ reporter. Error bars represent mean \pm s.e.m. c-e fib-1 knockdown induces $P_{i r g-1}$ GFP reporter and mRNA expression of irg-1. Error bars represent mean \pm s.e.m. $\mathbf{f}$ fib-1 RNAi reduces the levels of $26 \mathrm{~S}$ and $18 \mathrm{~S}$ rRNA in worms. RNA extracted from equal number of worms was analyzed using a bioanalyzer. $\mathbf{g}$, $\mathbf{h}$ fib-1 RNAi treatment reduced puromycin incorporation suggestive of reduced translation. No puromycin and cycloheximide treatments served as controls. Error bars represent mean \pm s.e.m. $\mathbf{i}$ ifg- 1 (cxTi9279) exhibits significantly extended survival compared to wild-type N2 upon S. aureus infection ( $P<0.0001$, log-rank test). j, k fib-1 knockdown significantly increases the survival of wild-type N2 ( $P<0.0001$, log-rank test) but not of ifg-1(cxTi9279) $(P=0.74$, log-rank test) upon $S$. aureus infection. Survival experiments were performed three times independently. Scale bars represent $100 \mu \mathrm{m} .{ }^{\star} P<0.05,{ }^{\star \star} P<0.01,{ }^{\star \star \star} P<0.001,{ }^{\star \star \star \star} P<0.0001$, ns - nonsignificant, unpaired $t$-test 
a

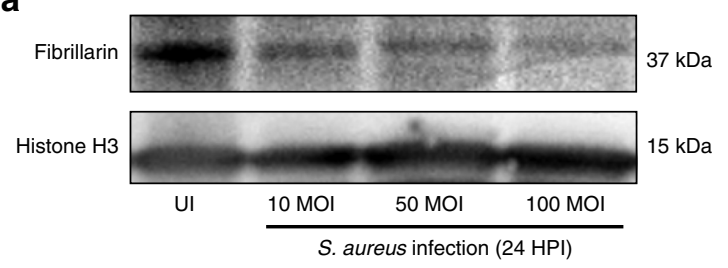

C

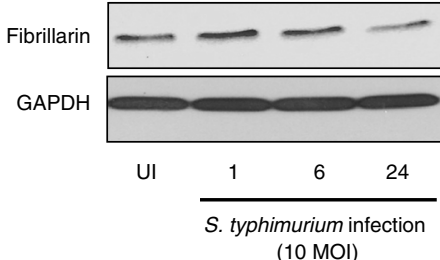

(10 MOI)

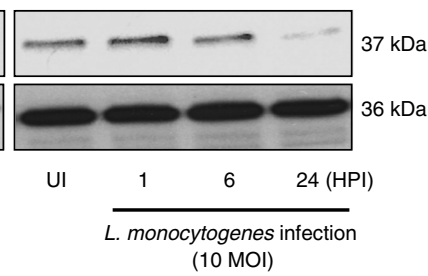

(10 MOI)

\section{b}

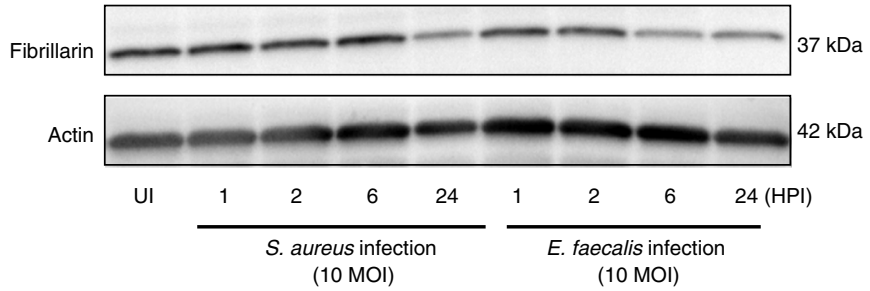

d

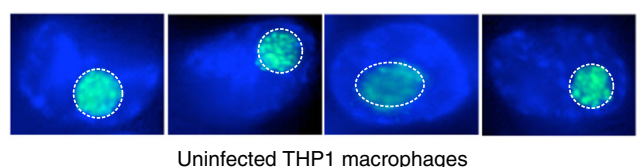

Uninfected THP1 macrophages

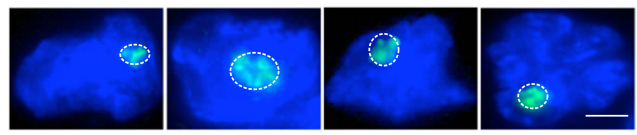

S. aureus infected THP1 macrophages (24 HPI)

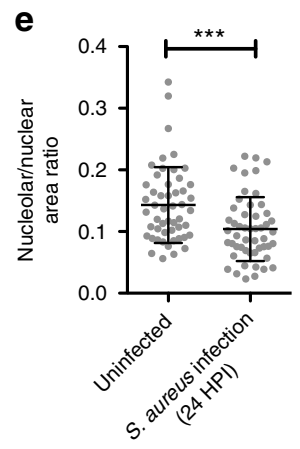

f

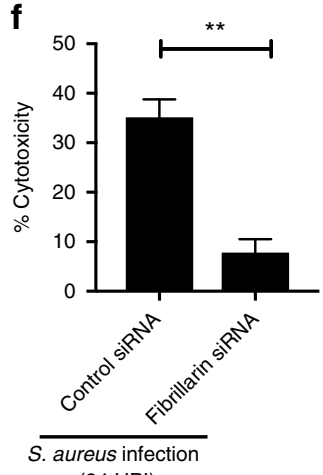

$(24 \mathrm{HPI})$

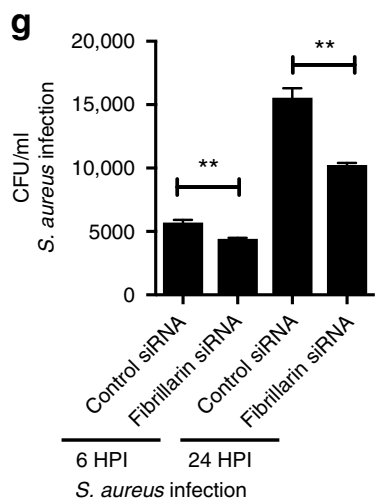

h

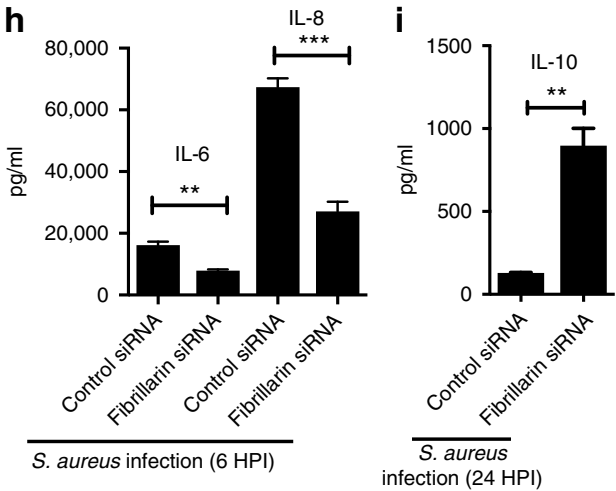

$\mathbf{k}$
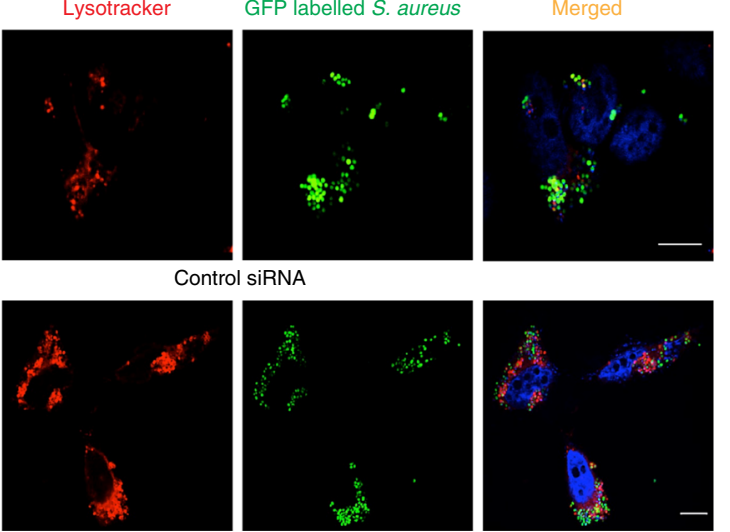

Fig. 5 Fibrillarin reduction protects mammalian cells against bacterial pathogens. a S. aureus infection leads to a reduction of fibrillarin levels in HeLa cells. b, c Mouse bone marrow-derived macrophages show a reduction in fibrillarin $24 \mathrm{~h}$ post infection with S. aureus, E. faecalis, S. typhimurium and L. monocytogenes. d, e Twenty-four hours of $S$. aureus infection leads to a reduction in nucleolar size of THP1 macrophages. Error bars represent mean \pm s.d. f Fibrillarin siRNA reduces cytotoxicity relative to control siRNA after $24 \mathrm{~h}$ of $\mathrm{S}$. aureus infection (MOI 10) in murine bone marrow-derived macrophages. Error bars represent mean \pm s.e.m., unpaired $t$-test. $\mathbf{g}$ Fibrillarin siRNA leads to a better clearance of intracellular pathogens thereby lowering the number of CFU per $\mathrm{mL}$ after 6 and $24 \mathrm{~h}$ of $\mathrm{S}$. aureus infection (MOI 10) in murine bone marrow-derived macrophages. Error bars represent mean \pm s.e.m., unpaired $t$-test. $\mathbf{h}$ Six hours after S. aureus infection, ELISA results show a decrease in pro-inflammatory cytokines interleukin 6 and 8 after fibrillarin siRNA treatment in HeLa cells. Error bars represent mean \pm s.e.m., unpaired $t$-test. i ELISA results show an increase in anti-inflammatory cytokine interleukin $10,24 \mathrm{~h}$ post infection with $S$. aureus upon fibrillarin knockdown in mouse bone marrow-derived macrophages. Error bars represent mean \pm s.e.m, unpaired $t$-test. j, k HeLa cells infected with GFP-labeled S. aureus and stained with lysotracker show increased co-localization of bacteria with lysosomes in cells treated with fibrillarin siRNA. ${ }^{\star \star} P<0.01,{ }^{\star \star \star} P<0.001$, unpaired $t$-test. Scale bars represent $4(\mathbf{d})$ and $10 \mu \mathrm{m}(\mathbf{j})$. UI uninfected, HPI hours post infection, MOI multiplicity of infection 
$P$. aeruginosa infection and conversely $n c l-1 /$ TRIM2 mutants that possess higher levels of fibrillarin ${ }^{14,24,25}$ are more susceptible to infection. Since C. elegans is largely dependent on innate immune signaling for pathogen resistance, the results indicate that higher levels of fibrillarin suppress innate immunity against bacterial pathogens. Interestingly, active bacterial infection and not heatkilled bacteria lead to a reduction in nucleolar size. This indicates that a reduction in nucleolar size and a decrease in fibrillarin levels constitute a host response mounted against live infection. $C$. elegans pathogen defense pathways are activated by a number of important factors including PMK-1/p38 MAPK, HLH-30/TFEB, and DAF-16/FOXO ${ }^{41}$. However, it still remains unclear how these different molecules coordinate downstream mechanisms to confer pathogen resistance. Our study suggests that fibrillarin regulates infection resistance as a convergent factor genetically downstream or parallel to these major players. How fibrillarin itself is regulated remains an interesting area of research to further pursue.

Pathogens often disrupt core cellular processes by delivering toxins so as to disable essential processes and pathways that would otherwise help mount a defense response. Multiple studies have reported that disruption of major cellular processes including mitochondrial respiration, proteasomal activity, microtubular dynamics, and mitochondrial unfolded protein response (UPR) can provoke immune-responsive genes, corroborating the notion of effector-triggered immunity $23,34,35,42$. Our work suggests a novel connection between the nucleolus and antibacterial immune function, and provides crucial evidence for a vital role of translation in imparting effector-triggered immunity. Improved infection resistance upon nucleolar and fibrillarin reduction likely works through translational control, since mutants that diminish translation trigger similar pathogen resistance, and fibrillarin knockdown only modestly improves survival of such mutants, suggesting overlapping mechanisms. Furthermore, we observe reduced levels of mature rRNA and translation upon fib-1 RNAi, adding more evidence that FIB-1 might orchestrate infection resistance via translational control. Surprisingly, fib-1 RNAi had little overt effect on global proteasome or autophagy activity, although it is conceivable that it could still affect these processes in a tissue or stage specific manner. While elegant studies have previously shown an induction of the immune response genes by reduced translation ${ }^{8}$, this work significantly extends these observations to demonstrate that this process broadly confers pathogen resistance. Since the nucleolus is involved in multiple cellular processes, however, it remains to be seen whether other nucleolar-regulated activities also influence immune function.

Fibrillarin is a highly conserved protein with similar structure and function in diverse species. Our study reveals a novel evolutionarily conserved function of fibrillarin in regulating innate immunity in mammals. Human epithelial cells and macrophages show a downregulation of fibrillarin after $24 \mathrm{~h}$ of infection, corroborating the results obtained in $C$. elegans and suggesting that reduction in fibrillarin levels as an ancestrally conserved hostdefense response toward infection. Depletion of fibrillarin dampened the secretion of pro-inflammatory cytokines and increased anti-inflammatory cytokines upon $S$. aureus infection, which also correlated with diminished cell death. Similarly, fibrillarin knockdown in C. elegans stimulated irg-1 expression but not a general transcriptional inflammatory response. This is consistent with the Damage Framework Model of Casadevall and Pirofski, which suggests that the severity of infection is guided by inflammation ${ }^{43}$. Inflammatory response to infection is required to defend against infection. However, overt inflammation makes the host susceptible to infection as a result of collateral damage to cells and tissues caused by the inflammatory cytokines. Therefore, pathogen resistance mechanisms encompass negative regulators of inflammation, which are required to elicit optimal host antimicrobial response. Moreover, fusion of the pathogen containing phagosome with lysosomes plays a vital role in containing infection, and inflammatory stimuli are also known to affect the process of phagosome maturation ${ }^{44}$. Consistently, we also observed an increased co-localization of intracellular bacteria with lysosomes in cells bearing fibrillarin knockdown. This intriguing observation points toward a possible role of fibrillarin in mediating lysosomal biogenesis or acidification, which needs to be further investigated. Whether the observed reduction in proinflammatory cytokine generation and increased cell survival is a result of accelerated phagosome maturation, translation regulation, or other processes affected by fibrillarin remains to be seen.

Our study opens multiple avenues to fundamentally explore the role of the nucleolus and fibrillarin in fighting pathogenic bacterial infections, and raises several important questions. What triggers fibrillarin downregulation upon infection? What is the mechanism by which this confers resistance? Might specific methylation sites in rRNA affect immune function? What are the dynamics of nucleolar and downstream processes driving pathogen resistance? With the current rise in antibiotic and multidrug resistant bacteria, future efforts toward the discovery of novel intrinsic cellular factors that hinder bacterial growth and improve host resistance may prove crucial. Conceivably, fibrillarin and related molecules could be used as vital targets for drug screens combating bacterial infections in mammals.

\section{Methods}

C. elegans strains. Worm strains were maintained at $20^{\circ} \mathrm{C}$ following standard procedures $^{45}$. N2 (wild type), ncl-1(e1865), ncl-1(e1942), daf-16(mu86), hlh-30 (tm1978), adIs2122(lgg-1::gfp; rol-6(su1006)), cguIs001 (FIB-1::GFP) ${ }^{46}$, ife-2 (ok306), ifg-1(cxTi9279), pmk-1(km25), agIs17 (myo-2p::mCherry + irg-1p::GFP).

Killing assay plate preparation. S. aureus (strain MW2-WT), E. faecalis (strain ATCC 29212), and P. aeruginosa (strain PA14) were used for infection in C. elegans. S. aureus was grown in tryptic soy broth medium. E. faecalis was grown in brain heart infusion (BHI) medium. P. aeruginosa was grown in lysogeny broth. Twenty microliters of saturated overnight bacterial cultures were spread on tryptic soy agar (TSA) with $10 \mu \mathrm{g} / \mathrm{mL}$ nalidixic acid (Sigma, NAL) for S. aureus, BHI with $10 \mu \mathrm{g} / \mathrm{mL}$ NAL for E. faecalis, and modified NGM, 0.35\% peptone, for P. aeruginosa. The plates were then incubated at $37^{\circ} \mathrm{C}$ overnight.

C. elegans killing assay. Age synchronized young adults were transferred to killing assay plates and the survival assay was carried out at $25^{\circ} \mathrm{C}$. For each condition, three technical replicates were set up with 20 worms on each plate. Scoring was performed every $12 \mathrm{~h}$ for $S$. aureus and P. aeruginosa and every $24 \mathrm{~h}$ for E. faecalis. Worms were scored as dead if they failed to respond to gentle touch with a worm pick. Animals that crawled off the plate or had vulval explosion were censored

qRT-PCR. Quantitative reverse transcription polymerase chain reaction (qRTPCR) was performed to measure RNA transcript levels. Age synchronized young adults were washed three times in M9 buffer and then transferred to TSA plates with $10 \mu \mathrm{g} / \mathrm{mL}$ nalidixic acid (Sigma, NAL) carrying either S. aureus or heat-killed $\mathrm{OP} 50$ at $25^{\circ} \mathrm{C}$. At the indicated times, animals were harvested and washed twice with M9 before lysis. Worms were lysed in QIAzol Lysis Reagent (Qiagen). RNA was isolated using RNeasy Mini kit (Qiagen). Complementary DNA synthesis was performed using iScript cDNA Synthesis Kit (Bio-Rad). Experiments were performed according to manufacturer's instructions. Quantitative PCR was performed on a ViiA 7 Real-Time PCR System (Applied Biosystems) using Power SYBR Green master mix (Applied Biosystems). All the experiments were performed three times independently and the results were normalized to $s n b-1$. qRT-PCR primer sequences are given in Supplementary Table 1. The primer sequences for pre-rRNA species were adopt from a recent study ${ }^{47}$

Western blotting. For worm western blot, 50 animals were transferred to killing assay plates and incubated at $25^{\circ} \mathrm{C}$ for $12 \mathrm{~h}$. Thirty animals from each condition were suspended in Laemmli lysis buffer with 5\% 2-mercaptoethanol (Sigma). For cells, $20 \mu$ g of protein were loaded on the gels. The samples were boiled at $95^{\circ} \mathrm{C}$ and loaded on Bio-Rad Midi-PROTEAN precast gels. Proteins were separated by standard sodium dodecyl sulfate-polyacrylamide gel electrophoresis (SDS-PAGE) and transferred to nitrocellulose membranes. The membranes were then blocked 
with $5 \%$ milk before probing with the following antibodies overnight: antifibrillarin (Novus Biologicals NB300-269, 1:1000), anti-Histone H3 (Abcam ab1791, 1:4000), anti- $\beta$ Actin (Abcam ab8224, 1:5000), anti-GAPDH (Santacruz sc47724, 1:2500), anti-puromycin (Millipore 12D10, 1:10000), anti-ubiquitin (Cell Signaling P4D1, 1:1000), anti-GFP (Takara 632381, 1:2000), anti-mouse HRP (ThermoFisher G-21040, 1:5000), anti-rabbit HRP (ThermoFisher G-21234, 1:5000). HRP means the antibodies are horseradish peroxidase conjugated. Uncropped versions of all the western blots referred to in the main text and figures are provided in Supplementary Figure 7.

Imaging and quantification. Differential interference contrast (DIC) microscopy was used to perform all the nucleolar imaging. Hypodermal cells of age-matched day 1 adults were imaged using $\times 100$ magnification with Olympus IX81. Freehand tool software from Fiji was used for nucleolar area quantification. FIB-1::GFP, HLH-30::GFP, and N2 $+\mathrm{P}_{\text {irg-1 }}$ GFP worms were imaged using Olympus IX81 and Axio Imager Z1 (Zeiss).

Puromycin incorporation assay. Worms treated with RNAi as described were incubated in S-basal medium supplemented with $0.5 \mathrm{mg} / \mathrm{mL}$ puromycin and OP50 as food source for $3 \mathrm{~h}$ at $20^{\circ} \mathrm{C}$ with gentle shaking. For the cycloheximide control, $2 \mathrm{mg} / \mathrm{mL}$ of the chemical was used. Thirty animals per condition were harvested for western blot detection of incorporated puromycin. The experiment was performed three times independently.

rRNA analysis. For infected samples, worms were treated the same as described for killing assay for $12 \mathrm{~h}$. Hundred animals were harvested for each sample. Worms were lysed and total RNA was obtained following the same protocol as performed for qRT-PCR. Total RNA samples, extracted from equal number of worms were analyzed on Agilent 2200 TapeStation System following the High Sensitivity RNA ScreenTape System protocol (Agilent). The experiment was performed three times independently.

Mammalian cell culture. Human epithelial cell lines, HeLa was obtained from ATCC. The cells were cultured in Dulbecco's modified Eagle's medium supplemented with $10 \%$ fetal calf serum (FCS). The cells were maintained at $37^{\circ} \mathrm{C}$ with $5 \% \mathrm{CO}_{2}$ in a humid atmosphere. The cells were tested for mycoplasma contamination.

Bone marrow-derived macrophages were prepared from 8-12 weeks old female C57BL/6J mice maintained and bred in the animal facility of Center for Molecular Medicine, University of Cologne, Germany. Mice were killed by cervical dislocation and bone marrows from the femurs were flushed using RPMI medium. The flushed cells were centrifuged and re-suspended in RPMI containing $10 \%$ FBS. Cells were seeded in culture dishes and allowed to differentiate into macrophages in medium supplemented with $20 \%$ L929 cell-culture supernatant for 7 days. Non-adherent cells were removed on days 2 and 4 , and adherent macrophages were used from day 7 onwards. Mouse experiments were performed according to the guidelines and approval of LANUV (Landesamt für Natur, Umwelt und Verbraucherschutz Nordrhein-Westfalen (State Agency for Nature, Environment and Consumer Protection North Rhine-Westphalia)).

THP1 monocytes were obtained from ATCC. The cells were tested for mycoplasma contamination. The cells were maintained in RMPI 1640 media, supplemented with $10 \%$ FCS. For differentiation of THP1 monocytes into macrophages, phorbol 12-myristate 13-acetate (PMA, Sigma, P8139) was used. Briefly, THP1 derived monocytes were incubated in 10\% FCS RPMI 1640 media supplemented with $25 \mathrm{ng} / \mathrm{mL}$ of PMA for $24 \mathrm{~h}$. The differentiated cells were used for infection.

Fibrillarin knockdown and overexpression. siGenome siRNA for fibrillarin was obtained from Dharmacon (GE Healthcare Life Sciences). The cells were treated with $100 \mathrm{nM}$ of siRNA $48 \mathrm{~h}$ before infection using Dharmafect-2 (GE Healthcare Life Sciences) according to the manufacturer's protocol. For mammalian plasmid transfection, plasmid carrying human fibrillarin fused to EGFP, cloned under CMV promoter (p-EGFP C1) was procured from Addgene (catalog no. 26673). The empty vector only carrying the EGFP was used as the control. HeLa cells were transfected with the plasmids using Lipofectamine 3000 (ThermoFisher Scientific) following the manufacturer's protocol. Transfected cells were assayed for fibrillarin and GFP expression $48 \mathrm{~h}$ post transfection and were then used for infection.

Infection in mammalian cells. A late logarithmic phase grown S. aureus (MW2), E. faecalis (ATCC 29212), S. Typhimurium (SL 1344), and L. monocytogenes (EGDe) were used at MOI 50 and MOI 10 for HeLa and macrophages, respectively. The cells were transfected with fibrillarin siRNA or overexpression plasmid for $48 \mathrm{~h}$ followed by infection. The infected cells were incubated for $10 \mathrm{~min}$ at room temperature, followed by an incubation for $30 \mathrm{~min}$ at $37^{\circ} \mathrm{C}$ with $5 \% \mathrm{CO}_{2}$ in a humid atmosphere. After $30 \mathrm{~min}$, extracellular bacteria were removed and cells were incubated for $2 \mathrm{~h}$ in medium containing $50 \mu \mathrm{g} / \mathrm{mL}$ gentamicin and then were washed and subsequently cultured in medium containing less gentamicin
$(10 \mu \mathrm{g} / \mathrm{mL})$. At desired time points cells were collected for western blot analysis using RIPA lysis buffer.

Cell viability assay. Cell viability was measured using a LDH Cytotoxicity Assay Kit (CytoTox 96 non-radioactive cytotoxicity assay; Promega). Released LDH was measured according to the manufacturer's protocol. The percentage of cell death was calculated using the formula: \% Cell death $=$ experimental release/maximum release $\times 100$. Trypan Blue method was performed by treating cells with trypsin at different time points post infection and counting viable cells using standard Trypan Blue dye exclusion assay.

Gentamycin protection assay. After S. aureus infection, the cells were washed three times with sterile PBS and lysed with $0.3 \%$ Triton X-100 in PBS for 5 min at room temperature. Several dilutions of the lysate were plated on BHI plates and incubated overnight at $37^{\circ} \mathrm{C}$. The following day, S. aureus $\mathrm{CFU}$ were counted.

Immunocytochemistry. HeLa cells were infected with GFP expressing S. aureus as per the above-mentioned protocol. Twenty-four hours post infection, the cells were incubated with $250 \mathrm{nM}$ lysotracker deep red (Invitrogen) for $15 \mathrm{~min}$ at $37^{\circ} \mathrm{C}$ with $5 \% \mathrm{CO}_{2}$ in a humid atmosphere. Then the cells were washed with warm PBS and fixed at room temperature for $15 \mathrm{~min}$ in $4 \%$ paraformaldehyde. Fixed cells were washed three times with PBS and mounted on slides with ProLong Gold mounting medium containing DAPI (ThermoFisher Scientific). Images were acquired with a $\times 60$ oil PlanApo objective numerical aperture 1.4 at room temperature on an Olympus IX81 inverted confocal microscope equipped with PMT detectors for imaging. Olympus Fluoview -10 ASW 4.2 software was used for acquisition and calculating Pearson's correlation.

ELISA. The supernatants from infected and uninfected cells were collected and snap frozen. ELISA was performed to gauge the levels of IL-6, IL-8, and IL-10 using DUOSet ELISA kits from R\&D Biosystems, following the manufacturer's protocol.

Blinding of experiments. All the C. elegans killing assays were performed in a blinded manner. For blinding, the bacterial strain and C. elegans strain names were concealed during scoring, analyzing, and plotting the data. Nucleolar imaging and quantification were also performed with concealed strain names. For blinding mammalian cell-culture experiments, one experimenter performed the siRNA transfections, and the experiment was blinded henceforth. The infections and other biochemical assays were carried out by the other experimenter.

\section{Data availability}

The authors declare that all the data and the methods used in this study are available within this article, its Supplementary Information files, the peer-review file, or are available from the corresponding author on request.

Received: 3 November 2017 Accepted: 19 July 2018

Published online: 06 September 2018

\section{References}

1. Mogensen, T. H. Pathogen recognition and inflammatory signaling in innate immune defenses. Clin. Microbiol. Rev. 22, 240-273 (2009).

2. Stuart, L. M., Paquette, N. \& Boyer, L. Effector-triggered versus patterntriggered immunity: how animals sense pathogens. Nat. Rev. Immunol. 13, 199-206 (2013).

3. Rajamuthiah, R. \& Mylonakis, E. Effector triggered immunity. Virulence 5, 697-702 (2014)

4. Iglewski, B. H., Liu, P. V. \& Kabat, D. Mechanism of action of Pseudomonas aeruginosa exotoxin Aiadenosine diphosphate-ribosylation of mammalian elongation factor 2 in vitro and in vivo. Infect. Immun. 15, 138-144 (1977).

5. Belyi, Y. et al. Legionella pneumophila glucosyltransferase inhibits host elongation factor 1A. Proc. Natl Acad. Sci. USA 103, 16953-16958 (2006).

6. Pacheco, A. R. \& Sperandio, V. Shiga toxin in enterohemorrhagic E. coli: regulation and novel anti-virulence strategies. Front. Cell. Infect. Microbiol. 2, 81 (2012).

7. Lee, M. S., Kim, M. H. \& Tesh, V. L. Shiga toxins expressed by human pathogenic bacteria induce immune responses in host cells. J. Microbiol. 51, 724-730 (2013)

8. Mohr, I. \& Sonenberg, N. Host translation at the nexus of infection and immunity. Cell Host Microbe 12, 470-483 (2012).

9. Jones, J. D. \& Dangl, J. L. The plant immune system. Nature 444, 323-329 (2006).

10. Gohre, V. \& Robatzek, S. Breaking the barriers: microbial effector molecules subvert plant immunity. Annu. Rev. Phytopathol. 46, 189-215 (2008).

11. Kleino, A. \& Silverman, N. UnZIPping mechanisms of effector-triggered immunity in animals. Cell Host Microbe 11, 320-322 (2012) 
12. Boisvert, F. M., van Koningsbruggen, S., Navascues, J. \& Lamond, A. I. The multifunctional nucleolus. Nat. Rev. Mol. Cell Biol. 8, 574-585 (2007).

13. Boulon, S., Westman, B. J., Hutten, S., Boisvert, F. M. \& Lamond, A. I. The nucleolus under stress. Mol. Cell 40, 216-227 (2010).

14. Tiku, V. et al. Small nucleoli are a cellular hallmark of longevity. Nat. Commun. 8, 16083 (2016).

15. Buchwalter, A. \& Hetzer, M. W. Nucleolar expansion and elevated protein translation in premature aging. Nat. Commun. 8, 328 (2017).

16. Hiscox, J. A. RNA viruses: hijacking the dynamic nucleolus. Nat. Rev. Microbiol. 5, 119-127 (2007).

17. Rodriguez-Corona, U., Sobol, M., Rodriguez-Zapata, L. C., Hozak, P. \& Castano, E. Fibrillarin from Archaea to human. Biol. Cell 107, 159-174 (2015).

18. Grummt, I. The nucleolus-guardian of cellular homeostasis and genome integrity. Chromosoma 122, 487-497 (2013).

19. Tessarz, P. et al. Glutamine methylation in histone $\mathrm{H} 2 \mathrm{~A}$ is an RNApolymerase-I-dedicated modification. Nature 505, 564-568 (2014).

20. Ermolaeva, M. A. \& Schumacher, B. Insights from the worm: the C. elegans model for innate immunity. Semin. Immunol. 26, 303-309 (2014).

21. Garsin, D. A. et al. Long-lived C. elegans daf-2 mutants are resistant to bacterial pathogens. Science 300, 1921 (2003).

22. TeKippe, M. \& Aballay, A. C. elegans germline-deficient mutants respond to pathogen infection using shared and distinct mechanisms. PLoS ONE 5, e11777 (2010).

23. Melo, J. A. \& Ruvkun, G. Inactivation of conserved C. elegans genes engages pathogen- and xenobiotic-associated defenses. Cell 149, 452-466 (2012).

24. Lee, L. W., Lee, C. C., Huang, C. R. \& Lo, S. J. The nucleolus of Caenorhabditis elegans. J. Biomed. Biotechnol. 2012, 601274 (2012).

25. Yi, Y. H. et al. A genetic cascade of let-7-ncl-1-fib-1 modulates nucleolar size and rRNA pool in Caenorhabditis elegans. PLoS Genet. 11, e1005580 (2015).

26. Hedgecock, E. M. \& Herman, R. K. The $n c l-1$ gene and genetic mosaics of Caenorhabditis elegans. Genetics 141, 989-1006 (1995).

27. Frank, D. J. \& Roth, M. B. ncl-1 is required for the regulation of cell size and ribosomal RNA synthesis in Caenorhabditis elegans. J. Cell Biol. 140, 1321-1329 (1998).

28. Arthur, J. S. \& Ley, S. C. Mitogen-activated protein kinases in innate immunity. Nat. Rev. Immunol. 13, 679-692 (2013).

29. Kim, D. H. et al. A conserved p38 MAP kinase pathway in Caenorhabditis elegans innate immunity. Science 297, 623-626 (2002).

30. Visvikis, O. et al. Innate host defense requires TFEB-mediated transcription of cytoprotective and antimicrobial genes. Immunity 40, 896-909 (2014).

31. Najibi, M., Labed, S. A., Visvikis, O. \& Irazoqui, J. E. An evolutionarily conserved PLC-PKD-TFEB pathway for host defense. Cell Rep. 15, 1728-1742 (2016).

32. Murphy, C. T. et al. Genes that act downstream of DAF-16 to influence the lifespan of Caenorhabditis elegans. Nature 424, 277-283 (2003).

33. McElwee, J., Bubb, K. \& Thomas, J. H. Transcriptional outputs of the Caenorhabditis elegans forkhead protein DAF-16. Aging Cell 2, 111-12 (2003).

34. McEwan, D. L., Kirienko, N. V. \& Ausubel, F. M. Host translational inhibition by Pseudomonas aeruginosa exotoxin A triggers an immune response in Caenorhabditis elegans. Cell Host Microbe 11, 364-374 (2012).

35. Dunbar, T. L., Yan, Z., Balla, K. M., Smelkinson, M. G. \& Troemel, E. R. C. elegans detects pathogen-induced translational inhibition to activate immune signaling. Cell Host Microbe 11, 375-386 (2012).

36. Estes, K. A., Dunbar, T. L., Powell, J. R., Ausubel, F. M. \& Troemel, E. R. bZIP transcription factor zip-2 mediates an early response to Pseudomonas aeruginosa infection in Caenorhabditis elegans. Proc. Natl Acad. Sci. USA 107, 2153-2158 (2010).

37. Syntichaki, P., Troulinaki, K. \& Tavernarakis, N. eIF4E function in somatic cells modulates ageing in Caenorhabditis elegans. Nature 445, 922-926 (2007).

38. Pan, K. Z. et al. Inhibition of mRNA translation extends lifespan in Caenorhabditis elegans. Aging Cell 6, 111-119 (2007).

39. Hansen, M. et al. Lifespan extension by conditions that inhibit translation in Caenorhabditis elegans. Aging Cell 6, 95-110 (2007).
40. Schnaith, A. et al. Staphylococcus aureus subvert autophagy for induction of caspase-independent host cell death. J. Biol. Chem. 282, 2695-2706 (2007).

41. Cohen, L. B. \& Troemel, E. R. Microbial pathogenesis and host defense in the nematode C. elegans. Curr. Opin. Microbiol. 23, 94-101 (2015).

42. Pellegrino, M. W. et al. Mitochondrial UPR-regulated innate immunity provides resistance to pathogen infection. Nature 516, 414-417 (2014).

43. Pirofski, L. A. \& Casadevall, A. The damage-response framework of microbial pathogenesis and infectious diseases. Adv. Exp. Med. Biol. 635, 135-146 (2008).

44. Pauwels, A. M., Trost, M., Beyaert, R. \& Hoffmann, E. Patterns, receptors, and signals: regulation of phagosome maturation. Trends Immunol. 38, 407-422 (2017).

45. Brenner, S. The genetics of Caenorhabditis elegans. Genetics 77, 71-94 (1974)

46. Lee, L. W., Lo, H. W. \& Lo, S. J. Vectors for co-expression of two genes in Caenorhabditis elegans. Gene 455, 16-21 (2010).

47. Zhou, X. et al. RdRP-synthesized antisense ribosomal siRNAs silence prerRNA via the nuclear RNAi pathway. Nat. Struct. Mol. Biol. 24, 258-269 (2017).

\section{Acknowledgements}

We thank C. elegans Genetic Center (CGC), University of Minnesota for providing C. elegans strains, Saray Gutierrez for help with ELISA experiments, and Jennifer Klimek for help with bacterial clearance experiments. This work was supported by the Max Planck Society, CECAD/Deutsche Forschungsgemeinschaft (DFG), and Cologne Graduate School of Ageing Research/DFG doctoral scholarship (V.T.).

\section{Author contributions}

V.T., C.K., P.M., N.R., and A.A. designed the study. V.T., C.K., P.M., R.G., and N.R. performed experiments and data analysis. V.T., P.M., N.R., and A.A. wrote the manuscript.

\section{Additional information}

Supplementary Information accompanies this paper at https://doi.org/10.1038/s41467018-06051-1.

Competing interests: The authors declare no competing interests.

Reprints and permission information is available online at http://npg.nature.com/ reprintsandpermissions/

Publisher's note: Springer Nature remains neutral with regard to jurisdictional claims in published maps and institutional affiliations.

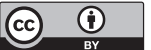

Open Access This article is licensed under a Creative Commons Attribution 4.0 International License, which permits use, sharing, adaptation, distribution and reproduction in any medium or format, as long as you give appropriate credit to the original author(s) and the source, provide a link to the Creative Commons license, and indicate if changes were made. The images or other third party material in this article are included in the article's Creative Commons license, unless indicated otherwise in a credit line to the material. If material is not included in the article's Creative Commons license and your intended use is not permitted by statutory regulation or exceeds the permitted use, you will need to obtain permission directly from the copyright holder. To view a copy of this license, visit http://creativecommons.org/ licenses/by/4.0/.

(C) The Author(s) 2018 